\title{
Arbeitsunfähigkeitsdauer und berufliche Rehabilitation nach arthroskopischer und offener Labrumrefixation
}

\author{
Duration of Inability for Work and Return to Physical Work \\ after Arthroscopic and Open Labrum Refixation
}

Autoren

Institut
A. Ateschrang*, S. Fiedler*, S. Schröter, U. Stöckle, T. Freude, T. M. Kraus

Klinik für Unfall- und Wiederherstellungschirurgie der Eberhard-Karls-Universität Tübingen, BG Unfallklinik Tübingen

\author{
Schlüsselwörter \\ - Schulterinstabilität \\ - Bankart-Läsion \\ - Labrumrefixation \\ - Schulterstabilisierung \\ - Instabilität \\ Key words \\ - shoulder instability \\ - Bankart lesion \\ - Bankart repair \\ - shoulder stabilisation \\ - instability
}

Bibliografie

DOI http://dx.doi.org/

10.1055/s-0034-1368407

Z Orthop Unfall 2014; 152:

252-259 ๑ Georg Thieme

Verlag KG Stuttgart - New York .

ISSN 1864-6697

Korrespondenzadresse

Dr. Atesch Ateschrang

Klinik für Unfall- und Wieder-

herstellungschirurgie der Eber-

hard-Karls-Universität Tübingen

BG Unfallklinik Tübingen

Schnarrenbergstraße 95

72076 Tübingen

Tel.: 07071/606-1166

Fax: 07071/606-1186

ate21@gmx.de

\section{Zusammenfassung \\ $\nabla$}

Hintergrund: Bisher gibt es keine Studie zur Arbeitsunfähigkeitsdauer (AU) in Abhängigkeit von der beruflichen Belastung nach offener und arthroskopischer Labrumrekonstruktion (LR). Ziel dieser Studie war daher der verfahrensspezifische Vergleich in Bezug auf die berufliche Tätigkeit nach REFA-Kriterien und der damit verbundenen Erfolgsrate beruflicher Rehabilitation.

Patienten und Methodik: Insgesamt wurden 93 Patienten $(20 \mathrm{w} / 73 \mathrm{~m})$ nach arthroskopischer und offener LR in diese retrospektive Studie eingeschlossen. 72 Patienten wurden arthroskopisch und 21 Patienten offen (Bankart/Neer) versorgt. Das klinische Follow-up beinhaltete den Constant-Murley, UCLA Shoulder sowie den Rowe Score. Das mittlere Follow-up betrug 48,3 Monate (Durchschnittsalter 37,1 Jahre). Die Arbeitsbelastung wurde nach REFA in $0-1$ (gering) und 2-4 (hoch) klassifiziert.

Ergebnisse: Arthroskopische LR (ALR): Die mittlere AU betrug 3,3 Monate $( \pm 2,5)$. Die AU für Patienten mit REFA $0-1$ betrug im Mittel 2,4 Monate $( \pm 1,6)$ und war signifikant kürzer als für Fälle mit REFA 2-4 mit 4,2 Monaten $( \pm 2,9 ; \mathrm{p}=0,0053)$. Zwei von 35 Patienten (5,7\%) mit REFA 0-1 und 10 von 37 Patienten (27,0\%) mit REFA 2-4 konnten ihre ursprüngliche Tätigkeit nicht wieder aufnehmen. Offene LR (OLR): Die mittlere AU betrug 2,7 Monate $( \pm 2,3)$. Die AU betrug für Patienten mit REFA 0-1 durchschnittlich 1,8 Monate $( \pm 1,0$ Monate) und war kürzer als für Fälle mit REFA 24 mit 4,3 Monaten $( \pm 3,3 ; p=0,1196)$. Einer aus 14 Patienten mit REFA 0-1 (7,1\%) und 4 aus 7 Patienten $(57,1 \%)$ mit REFA 2-4 konnten ihre ursprüngliche Tätigkeit nicht wieder vollständig aufnehmen. Es fanden sich keine verfahrensbedingten

\section{License terms}

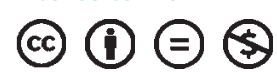

* Beide Autoren haben zu gleichen Teilen zur Entstehung dieser Arbeit beigetragen.

\section{Abstract \\ $\nabla$}

Background and Introduction: The duration of inability for work according to work load and the rate of successful return to work after open and arthroscopic Bankart repair (BR) due to anterior shoulder dislocation has not yet been examined with regard to validated work strain by the REFA classification. Thus, the objective of this study was to determine the duration of inability to work according to work load (REFA criteria) after open and arthroscopic BR as well as the rate of successful return to the original occupation.

Patients and Methods: A total of 93 patients $(20 \mathrm{f} / 73 \mathrm{~m})$ with isolated anterior arthroscopic or open BR due to posttraumatic anterior shoulder instability with no items of hyperlaxity were included in this study. There were 72 patients with arthroscopic and 21 patients with open BR. The postoperative aftercare was standardised and identical. The clinical assessment included the Constant and Murley scores, UCLA shoulder and the Rowe score for shoulder instability. Average follow-up time was 48.3 months (SD \pm 23.6 months) with a mean age of 37.1 years (SD \pm 14.4 years). The work load was classified according to the German REFA Association. Operation time, duration of inability for work and clinical outcome were analysed and compared according to the operation technique.

Results: Mean incapacity for work in the group of arthroscopic BR was 3.3 months (SD \pm 2.5$)$ and 2.7 months (SD +2.3 months; $p=0.37$ ) in the group of open BR demonstrating no statistical difference. Both mean time for surgery $(p=0.0003)$ and inhospital stay $(\mathrm{p}=0.0083$ ) showed significant differences when comparing patients with low work load (REFA 0-1) and higher work load (REFA 2-4) irrespective of the surgical approach. Overall analysis showed an average time of 2.3 months ( $S D \pm 1.5)$ to return to work for patients with low work load (REFA 0-1) and 4.2 months (SD \pm 2.9 ) 
Unterschiede hinsichtlich des Vergleichs zwischen den REFAspezifischen Subgruppen nach offener und arthroskopischer LR. Insgesamt konnten 18,3\% aller Patienten ihre ursprüngliche Arbeit nicht mehr ausüben.

Schlussfolgerung: Höhere Arbeitsbelastungen (REFA 2-4) führen zu signifikant längerer $\mathrm{AU}$, sowohl nach offener wie arthroskopischer LR, wobei keine verfahrensbedingten Vorteile vorliegen. Das arthroskopische und offene Vorgehen zeigten klinisch äquivalente Ergebnisse. Die primäre arthroskopische LR kann auch für Betroffene mit belastender Tätigkeit empfohlen werden, wobei die berufliche Rehabilitationsprognose verbesserungswürdig ist. for individuals with high work load (REFA 2-4) revealing significant differences $(\mathrm{p}=0.0006)$. The mean inability for work after arthroscopic BR for patients with REFA 0-1 was 2.4 months (SD $\pm 1.6)$ and 4.2 months $( \pm 2,9 ; p=0.0053)$ for patients with REFA 2-4 revealing a significant difference. The mean inability for work after open BR for individuals with REFA $0-1$ was 1.8 months $( \pm 1.0)$ and 4.3 months $( \pm 3.3 ; \mathrm{p}=0.1196)$ for individuals with REFA 2-4. Two out of 35 patients (5.7\%) with low work load (REFA $0-1$ ) and 10 out of 37 patients (27\%) with high work load (REFA 2-4) could not return to their original occupation after arthroscopic BR. One out of 14 patients $(7.1 \%)$ with low work load (REFA 0-1) and 4 out of 7 patients (57.1\%) with high work load (REFA 2-4) could not return to their original occupation after open BR. Comparing these results between arthroscopic and open BR, no significant differences were obtained (chi-square, Pearson). Recurrent shoulder dislocation occurred in the arthroscopic group in $14.3 \%$ (REFA 0-1) and 8.1\% (REFA 2-4) compared to the open procedure group in 0\% (REFA 0-1) and 14\% (REFA 24) revealing no statistically significant differences.

Conclusion: This study showed equivalent results after performing open and arthroscopic BR with significantly shorter operation times and in-hospital stays after arthroscopic BR. Higher work loads caused longer inability for work irrespective of the chosen surgical technique. On the basis of these results we recommend arthroscopic BR as the standard primary procedure, while the overall rate of return to work without restrictions of $81.7 \%$ has to be improved in the future.

\section{Einleitung}

\section{$\nabla$}

Die Inzidenz traumatisch bedingter anteriorer Schulterinstabilität wird zwischen 0,023\% [1] und 1,7\% [2] geschätzt und spielt damit im klinischen Alltag eine relevante Rolle. Traumatische Schulterluxationen können zu Läsionen des Labrums [3], der Rotatorenmanschette, des Glenoids [4] sowie der kapsuloligamentären Strukturen führen [5].

Historisch wurde die offene Bankart-Operation [6] zur Rekonstruktion von Labrumläsionen bereits 1938 vom gleichnamigen Autor etabliert und fortlaufend durch technische Verbesserungen der Rekonstruktionstechnik modifiziert. Die damit erzielten Ergebnisse der offenen Bankart-Operation wurden in einer Vielzahl von Studien publiziert [7-9]. In den vergangenen 15 Jahren wurde die offene Operation zunehmend durch arthroskopische Verfahren verdrängt. Während vor allem zu Beginn eine erhöhte Reluxationsrate gegen das arthroskopische Vorgehen sprach, konnten durch zunehmende Erfahrungen und Verbesserung der arthroskopischen Technik äquivalente Ergebnisse bei reduziertem Operationstrauma erzielt werden. Metaanalysen der letzten Jahre bescheinigten der offenen und arthroskopischen Stabilisierung gute langfristige Ergebnisse $[10,11]$. Weitere Studien zeigten, dass durch technische Verbesserungen des arthroskopischen Vorgehens auch ein besseres Outcome erzielt werden konnte [12, 13].

Während früher Glenoiddefekte der offenen Operation vorbehalten waren, kann dank verbesserter Technik und Erfahrung des Chirurgen immer öfter das arthroskopische Vorgehen realisiert werden $[14,15]$. Das offene Verfahren behält aber seinen Stellenwert, bspw. bei rezidivierenden Luxationen, insbesondere in weniger spezialisierten Institutionen $[16,17]$.

Während die Ergebnisse nach offener und arthroskopischer Labrumrekonstruktion für Sportler untersucht wurden [4,18-23] gibt es keine uns bekannte Studie mit Analyse der erfolgreichen beruflichen Rehabilitation sowie unter Berücksichtigung der Arbeitsbelastung nach den Kriterien der Deutschen Rentenversicherung, der REFA-Klassifikation.

Ziel dieser Studie war daher, zu analysieren, wie hoch die jeweilige Rate der Patienten nach arthroskopischer und offener Labrumrekonstruktion (LR) war, die ihre ursprüngliche berufliche Tätigkeit unter besonderer Berücksichtigung der REFA-Klassifikation wieder aufnehmen konnten und der damit vergesellschafteten Reluxationsraten.

\section{Patienten und Methoden \\ $\nabla$}

In diese retrospektive Studie wurden 93 Patienten nach offener (2003-2005) und arthroskopischer (2005-2012) Bankart-Operation eingeschlossen. Einschlusskriterien waren die bestehende Arbeitsfähigkeit vor Verletzung und ein Alter von 18 bis 65 Jahren sowie die unilaterale posttraumatische anteriore Schulterinstabilität definiert. Als Ausschlusskriterien galten begleitende Tuberculum-majus-Frakturen, eine koinzidente hyperlaxe Instabilitätskomponente sowie Rotatorenmanschettenläsionen. Patienten mit Glenoidfrakturen, knöcherner Bankart-Läsion oder mit ausgedehnten Knorpelschäden wurden ebenso ausgeschlossen. Bis 2005 erfolgte in unserer Institution die Schulterstabilisierung nach Bankart routinemäßig offen und wurde im Zuge der Etablierung der arthroskopischen Technik konvertiert auf das geschlossene Vorgehen. $\triangle$ Abb. 1 gibt einen Überblick über das gesamte Patientengut unter Berücksichtigung der Ein- und Ausschlusskriterien.

Neben der Einteilung in eine offene und arthroskopische Therapiegruppe wurde die Arbeitsbelastung nach REFA-Kriterien klassifiziert ( Tab. 1). Für verbesserte Übersichtlichkeit wurden die 
Tab. 1 Klassifikation der Arbeitsbelastung nach REFA-Leitlinien (nach [31]).

\begin{tabular}{|lll}
$\begin{array}{l}\text { Grad } \\
0\end{array}$ & $\begin{array}{l}\text { Arbeitsbelastung } \\
\text { Arbeit ohne spezielle körperliche Beanspruchung }\end{array}$ & $\begin{array}{l}\text { Beispiel } \\
\text { Arbeit ohne körperliche Belastung, wie z. B. reine Schreibtischarbeit }\end{array}$ \\
\hline 1 & Arbeit mit geringer körperlicher Beanspruchung & Bearbeitung von leichten Werkgegenständen; ebenso langes Stehen oder häufiges Gehen \\
\hline 2 & Arbeit mit mittlerer körperlicher Beanspruchung & $\begin{array}{l}\text { Bedienung von Geräten zwischen } 1 \text { und } 3 \mathrm{~kg} \text {; Tragen von Lasten zwischen } 10 \text { und } 15 \mathrm{~kg} \text {; Treppen- } \\
\text { steigen oder Steigen von Leitern ohne zusätzliche Last }\end{array}$ \\
\hline 3 & Arbeit mit schwerer körperlicher Beanspruchung & $\begin{array}{l}\text { Tragen von Lasten zwischen } 20 \text { und } 30 \mathrm{~kg} \text {; Schaufel-arbeiten, Graben, Holzarbeiten, Treppensteigen } \\
\text { oder Steigen von Leitern mit mittlerer Tragelast, mäßige Arbeit in angespannter Körperhaltung }\end{array}$ \\
\hline 4 & $\begin{array}{l}\text { Arbeit mit schwerster körperlicher Beanspru- } \\
\text { chung }\end{array}$ & $\begin{array}{l}\text { Tragen von Lasten mit mehr als } 50 \mathrm{~kg} \text {, Treppensteigen oder Steigen von Leitern mit schwerer } \\
\text { Tragelast, schwere Arbeit in angespannter Körperhaltung }\end{array}$ \\
\hline
\end{tabular}

REFA-Gruppen 0-1 (niedrige Arbeitsbelastung) und REFA-Gruppen 2-4 (hohe körperliche Belastung) zusammengefasst. Das klinische Ergebnis wurde in puncto Funktion und Stabilität nach dem UCLA Score [24], dem Rowe Score [9] sowie dem Constant and Murley Score [25] beurteilt. Die Dauer der Arbeitsunfähigkeit und die berufliche Rehabilitation in die ursprüngliche Tätigkeit (zum Zeitpunkt der Verletzung) wurden protokolliert.

Diese Studie wurde durch die zuständige Ethikkommission berufsrechtlich geprüft.

\section{Arthroskopische Bankart-Rekonstruktion}

Die Operation erfolgte standardisiert unter Vollnarkose in Beachchair-Lagerung des Probanden. Der Eingriff erfolgte mit 3 Arthroskopieportalen: Zu Beginn erfolgte die diagnostische Arthroskopie, um weitere koinzidente Begleitverletzungen auszuschließen oder zu bestätigen. Arthroskopisch assistiert wurden jeweils ein anterior-superiorer sowie ein anterior-inferiorer Zugang mit Arbeitstrokaren angelegt. Anschließend wurden der abgelöste Kapsel-Labrum-Komplex mobilisiert sowie die Knochenoberfläche mittels Shaver angefrischt, um eine dauerhafte orthotope knöcherne Einheilung des Kapsel-Labrum-Komplexes zu ermöglichen. Zwei bis 3 Bio-Fastak-Fadenanker $\left(\right.$ Arthrex $\left.^{\circledR}\right)$ mit einer Dimension von $3 \times 14 \mathrm{~mm}$ wurden am Glenoidvorderrand platziert. Das Fadenmaterial wurde unter Nutzung von Shuttle-Instrumenten durch den mobilisierten Kapsel-Labrum-Komplex gestochen, um die anatomische Reposition des Kapsel-Labrum-Komplexes zu erzielen. Die $\triangle$ Abb. 2 und $\mathbf{3}$ verdeutlichen dieses Vorgehen anhand eines Beispiels.

\section{Offene Stabilisierung nach Bankart und Neer}

Die offene Stabilisierung nach Bankart und Neer erfolgte standardisiert in Beachchair-Lagerung und Intubationsnarkose. Hierzu wird ein klassischer deltoideopektoraler Zugang angelegt. Nach Spaltung der Subkutis wird der Sulcus deltoideopectoralis mit der nach lateral verlagerten $\mathrm{V}$. cephalica auseinandergedrängt und anschließend die Fascia clavipectoralis gespalten. Die Subskapularissehne wurde ca. $1 \mathrm{~cm}$ medial der Insertionsstelle intratendinös durchtrennt, von der darunterliegenden Gelenkkapsel separiert und mit Haltefäden gesichert. Die Gelenkkapsel wurde separat eröffnet und auch mit Haltefäden armiert, gefolgt von der Beurteilung des Kapsel-Labrum-Komplexes sowie der Gelenkund Knorpeloberfläche. Das Labrum glenoidale wurde mit dem adhärenten Kapsel-Band-Komplex mobilisiert und die knöcherne Glenoidvorderfläche angefrischt. An die Glenoidvorderkante wurden 2 bis 3 Bio-Fastak-Fadenanker (Arthrex ${ }^{\circledR}$ ) eingebracht, um das Labrum an anatomischer Position zu fixieren. Anschließend wurde die Gelenkkapsel nach Neer gerafft und die Subskapularissehne intratendinös vernäht.

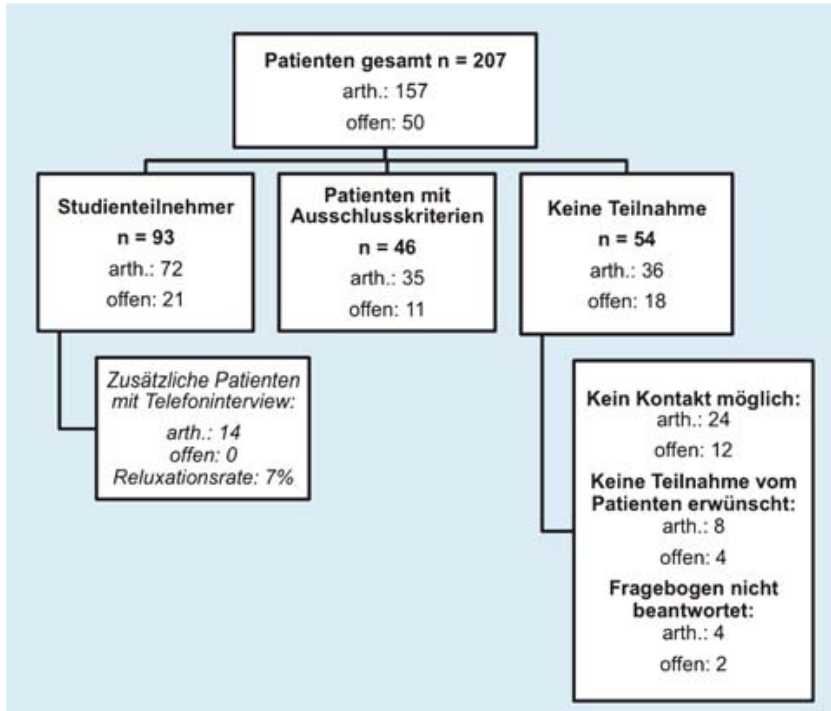

Abb. 1 Zusammensetzung des gesamten Patientenkollektivs.

\section{Postoperativer Behandlungsplan}

Die postoperative Nachbehandlung erfolgte für die arthroskopisch und offen rekonstruierten Schultergelenke identisch: Ab dem 1. postoperativen Tag wurde das Schultergelenk des Patienten mit einem Ultrasling-Abduktionskissen (DJO Global, Deutschland) immobilisiert. Dabei wurde das Gelenk in $0^{\circ}$ Außenrotation ruhiggestellt. Für die folgenden 10 Tage wurde die Schulter passiv zwischen 30 und $60^{\circ}$ in Anteversion und Abduktion bewegt. Die Außenrotation im Schultergelenk wurde für 6 Wochen postoperativ vermieden. $\mathrm{Ab}$ dem 11 . postoperativen Tag konnte unter kontrollierten Bedingungen $90^{\circ}$-Abduktion und Anteversion realisiert werden. Das Ultrasling-Abduktionskissen wurde ab diesem Zeitpunkt nur nachts getragen. Ab der 7. postoperativen Woche konnte die Schulter ohne weitere Einschränkungen im Alltag eingesetzt werden. Es wurde Sportkarenz (Wettkampfsport sowie Überkopfsportarten) für 6 Monate postoperativ empfohlen.

\section{Statistische Analyse}

Die statistische Auswertung der Daten erfolgte mit JMP 9.0 für Mac (SAS Institute Inc., Cary, North Carolina, USA). Die Ergebnisse des UCLA Shoulder Scores, des Rowe Scores sowie des Constant and Murley Scores wurden mittels Welch-Test sowie ANOVA verglichen. Die Arbeitsunfähigkeitsdauer wurde mittels ANOVA-tTest analysiert. Die angegebenen Werte wurden als geometrische Mittelwerte errechnet. Die verfahrensspezifischen Luxationsrezidivraten wurden mittels Chi-Quadrat-Test nach Pearson vergli- 

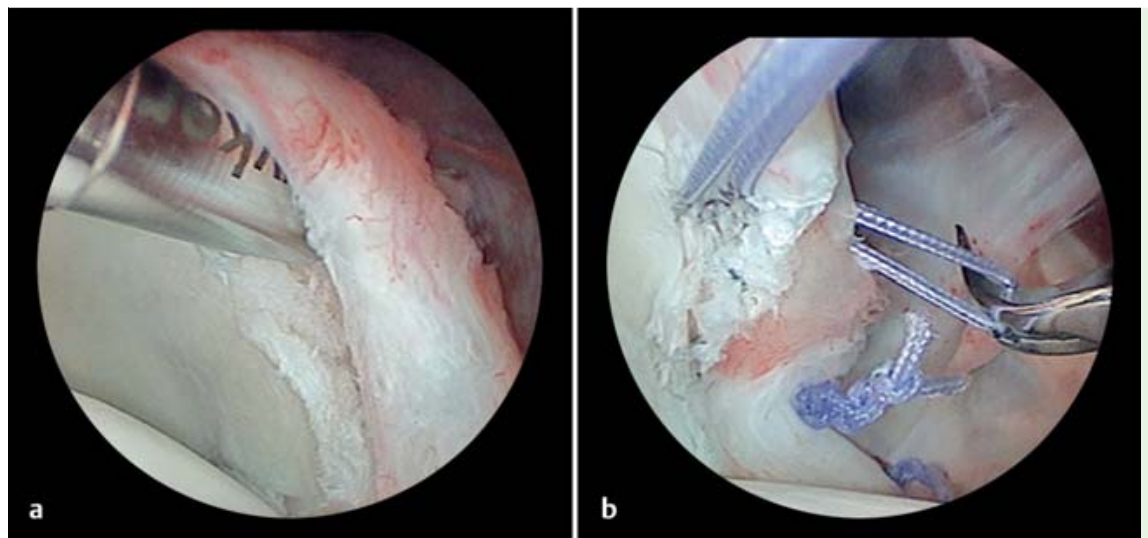

Abb. 2 a und $\mathbf{b}$ a Intraoperativer Situs mit Débridement der Glenoidvorderfläche und des Scapulahalses mittels Shaver. b Intraoperative Situation bei Labrumrefixation mit 3 Fadenankern.

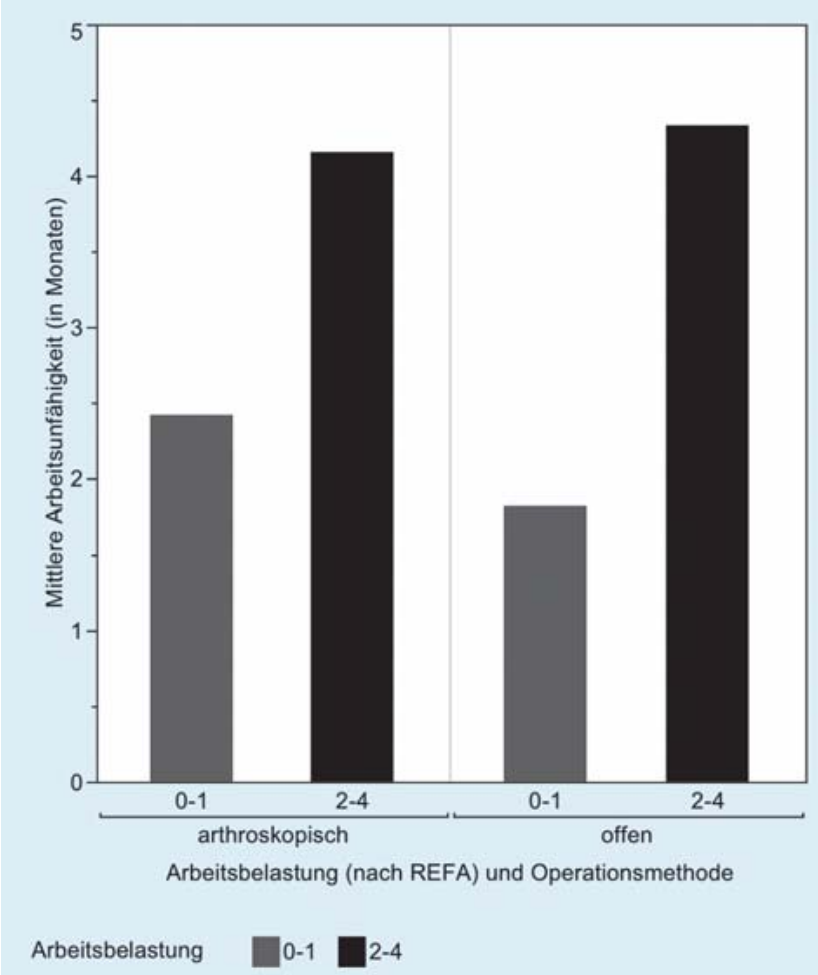

Abb. 3 Arbeitsunfähigkeitsdauer (in Monaten) in Abhängigkeit von der Arbeitsbelastung (REFA 0-1: schwarze Balken, REFA 2-4: graue Balken) und der Operationsmethode (arthroskopisch vs. offen).

chen. Der jeweils angewandte statistische Test wurde in den Ergebnistabellen angegeben. Das Signifikanzniveau wurde auf $p=0,05$ festgelegt. Die statistische Auswertung erfolgte unter Supervision des lokalen Instituts für klinische Epidemiologie und angewandte Biometrie.

\section{Ergebnisse \\ $\nabla$}

Insgesamt wurden 73 Männer und 20 Frauen nachuntersucht, die in den Jahren 2003 bis 2012 operiert worden waren. Das Durchschnittsalter betrug zum Nachuntersuchungszeitpunkt 37,1 Jahre $(S D \pm 14,4)$. Die mittlere Zeit zwischen Operation und Nachuntersuchung betrug 48,3 Monate (SD $\pm 23,6$ ). Die mittlere
Operationsdauer betrug $60,5 \mathrm{~min}$ ( $\mathrm{SD} \pm 28,2$ ). Die mittlere stationäre Verweildauer $4,7 \mathrm{~d}(\mathrm{SD} \pm 2,5)$. Die Reluxationsrate betrug $9,7 \%$ für das gesamte Patientenkollektiv.

\section{Klinische Gesamtergebnisse}

Die Betrachtung aller Patienten ergab eine mittlere Dauer der Arbeitsunfähigkeit von 3,2 Monaten (SD $\pm 2,5$ ). Die klinischen $\mathrm{Er}$ gebnisse waren für das gesamte Patientenkollektiv als gut zu bewerten mit einem mittleren UCLA Shoulder Score von 31,5 (SD $\pm 4,2$ ), einem durchschnittlichen Rowe Score von 86 (SD $\pm 21,8$ ) und einem mittleren Constant and Murley Score von 86,2 (SD $\pm 14,3)$. @ Tab. 2 gibt eine Übersicht.

\section{Ergebnisse nach offener vs. arthroskopischer Bankart-Operation}

Die durchschnittliche Operationsdauer für das arthroskopische Vorgehen betrug 53,9 $\min (S D \pm 24,1)$ und $94,3 \min (S D \pm 23,8)$ für das offene Vorgehen mit einem resultierenden signifikanten Unterschied ( $p=0,0003$, t-Test). Die stationäre Verweildauer betrug nach ALR 4,3 d $(S D \pm 2,2)$ im Vergleich zu 6,3 d (SD $\pm 2,3)$ nach OLR, wobei sich ein signifikanter Unterschied zeigte $(p=0,0083, t-T e s t)$. Die Arbeitsunfähigkeitsdauer lag nach ALR bei 3,3 Monaten (SD $\pm 2,5$ ) und nach OLR bei 2,7 Monaten (SD $\pm 2,3$ ). Hierbei zeigte sich kein signifikanter Unterschied. Für Fälle nach ALR zeigte sich eine Reluxationsrate von $11,1 \%(n=8)$ vs. $4,8 \%(n=1)$ nach OLR ohne statistisch signifikanten Unterschied ( $p=0,46$, Chi-Quadrat nach Pearson). Die postoperativen Ergebnisse ergaben für die erwähnten Scores keine signifikanten Unterschiede. Die einzelnen Ergebnisse sind in O Tab. $\mathbf{3}$ und Abb. $\mathbf{4}$ übersichtlich dargestellt.

\section{Operationszeit, stationäre Verweildauer sowie Reluxati- onsrate}

Vergleicht man die Operationsdauer unabhängig von der Operationsmethode sondern in Abhängigkeit von der körperlichen Arbeitsbelastung des Patienten, zeigte unsere Auswertung Operationszeiten mit durchschnittlich $66,2 \mathrm{~min}(\mathrm{SD} \pm 32,5)$ für Patienten der Kategorie REFA 0-1 und im Mittel 53,3 min (SD $\pm 20,0)$ für Patienten der Kategorie REFA 2-4, ohne dabei signifikant zu sein $(p=0,062$, $t$-Test). Der Vergleich der stationären Verweildauer zeigte für REFA-Gruppen 0-1 eine mittlere Behandlung von $4,1 \mathrm{~d}(\mathrm{SD} \pm 1,8)$ gegenüber $5,4 \mathrm{~d}(\mathrm{SD} \pm 2,9)$ für REFA-Gruppen 2-4 mit daraus resultierendem signifikantem Unterschied $(p=0,0091$, t-Test). Die Analyse der Arbeitsunfähigkeitsdauer zeigte signifikant ( $p=0,0006$, t-Test) längere Ausfallzeiten für Patienten mit hoher körperlicher Arbeitsbelastung (REFA 2-4: 4,2 
Tab. 2 Klinische Ergebnisse nach offener und arthroskopischer Rekonstruktion.

$\begin{array}{lllllll}\mathbf{n} & \begin{array}{l}\text { Alter in Jahren } \\ (\mathbf{M W} \pm \mathbf{S D})\end{array} & \begin{array}{l}\text { Dauer bis zur Nach- } \\ \text { untersuchung in } \\ \text { Monaten }(\mathbf{M W} \pm \text { SD) }\end{array} & \begin{array}{l}\text { Arbeitsunfähigkeit } \\ \mathbf{( M W} \pm \mathbf{S D}) \text { in } \\ \text { Monaten }\end{array} & \begin{array}{l}\text { UCLA Shoulder } \\ \text { Score (MW } \pm \text { SD) }\end{array} & \begin{array}{l}\text { Rowe Score } \\ \text { (MW SD) }\end{array} & \begin{array}{l}\text { Constant and } \\ \text { Murley Score } \\ \text { (MW } \pm \text { SD) }\end{array} \\ 93 & 37,1 \pm 14,4 & 48,3 \pm 23,6 & 3,18 \pm 2,48 & 31,5 \pm 4,2 & 86 \pm 21,8 & 86,2 \pm 14,3\end{array}$

Monate $\pm 2,9$ ) gegenüber Patienten mit geringer körperlicher $\mathrm{Ar}$ beitsbelastung (REFA 0-1: 2,3 Monate $\pm 1,5$ ).

Die Reluxationsraten erbrachten mit 10,2\% in den REFA-Gruppen $0-1$ und 9,1\% in den REFA-Gruppen 2-4 keine signifikanten Unterschiede ( $p=0,86$; Chi-Quadrat nach Pearson). Während sich beim UCLA Shoulder Score mit 32,6 (SD $\pm 3,4$ ) in den REFA-Gruppen $0-1$ und $30,4(S D \pm 4,8)$ ein signifikanter Unterschied $(\mathrm{p}=0,014$, t-Test) zeigte, wurden beim Rowe Score und beim Constant and Murley Score keine signifikanten Differenzen festgestellt. Eine zusammenfassende Aufstellung dieser Ergebnisse ist $\bigcirc$ Tab. 4 zu entnehmen.

\section{Arbeitsunfähigkeit und berufliche Rehabilitation}

Eine weitere Analyse der Ergebnisse unterteilt nach Subgruppen (spezifisch nach Operationsmethode mit leichter und schwerer körperlicher Arbeitsbelastung), ist $\odot$ Tab. 5 zu entnehmen. Signifikante längere Arbeitsunfähigkeitsintervalle wurden für Probanden mit körperlich belastender Tätigkeit (REFA 2-4) nach arthroskopischer Rekonstruktion mit 4,2 Monaten (SD $\pm 2,9$ ) gegenüber 2,4 Monaten (SD $\pm 1,6)$ mit geringer körperlicher Belastung (REFA 0-1) festgestellt ( $p=0,0053$, t-Test). Nach offener Labrumrekonstruktion zeigten sich auch signifikant längere Arbeitsunfähigkeitsintervalle für Probanden mit körperlich belastender Tätigkeit (REFA 2-4) mit 4,3 Monaten (SD $\pm 3,3$ ) gegenüber 1,8 Monaten $(S D \pm 1,0)$ für Patienten mit geringer Belastung (REFA 0-1) ( $\mathrm{p}=0,1196)$.

Werden die verfahrensspezifischen Gruppen (ALR vs. OLR) unter Berücksichtigung der beruflichen Belastung (REFA 0-1 vs. REFA 2-4) verglichen, konnten sowohl zwischen den REFA-Gruppen 0-1 ( $\mathrm{p}=0,1590)$ als auch zwischen den REFA-Gruppen 2-4 $(\mathrm{p}=0,9052)$ keine signifikanten Unterschiede festgestellt werden. Bezüglich der Reluxationsrate ergab die statistische Kalkulation mit dem Chi-Quadrat-Pearson-Test sowohl bei gleicher Operationstechnik und unterschiedlicher Arbeitsbelastung als auch bei gleicher Arbeitsbelastung und unterschiedlicher Operationsmethode keine Unterschiede ( Tab. 5). Gleiches gilt für die klinische Beurteilung durch die oben genannten Scores. Hier zeigten sich nur beim UCLA Shoulder Score zwischen arthroskopisch operierten Patienten mit REFA 0-1 (32,9 $\pm 2,5)$ und REFA 2-4 (30,6 \pm $5,1)$ signifikante Unterschiede ( $\mathrm{p}=0,0140$, $\mathrm{t}$-Test). Zwei von $35 \mathrm{~Pa}-$ tienten $(5,7 \%)$ mit REFA $0-1$ und 10 von 37 Patienten (27\%) mit REFA 2-4 konnten ihre ursprüngliche Tätigkeit nach arthroskopischer LR nicht wieder aufnehmen. Nach offener Labrumrekonstruktion konnte 1 Proband aus 14 Patienten mit REFA 0-1 (7,1\%) und 4 aus 7 Patienten (57,1\%) mit REFA 2-4 ihre ursprüngliche Tätigkeit nicht wieder vollständig wie ursprünglich aufnehmen (Umsetzung des Arbeitsplatzes). $\odot$ Tab. 5 gibt einen Überblick.

Ein Teil der Patienten $(n=14)$ konnte nicht an der Nachuntersuchung teilnehmen, sie wurden aber telefonisch über ihre aktuelle Beschwerdesymptomatik befragt. Hiervon gaben 3 Patienten Restbeschwerden an. Ein Patient klagte weiterhin über eine bestehende Restinstabilität. Die restlichen Patienten (11) hatten keine Beschwerden und konnten ihre Schulter wieder ohne relevante Einschränkungen einsetzen.

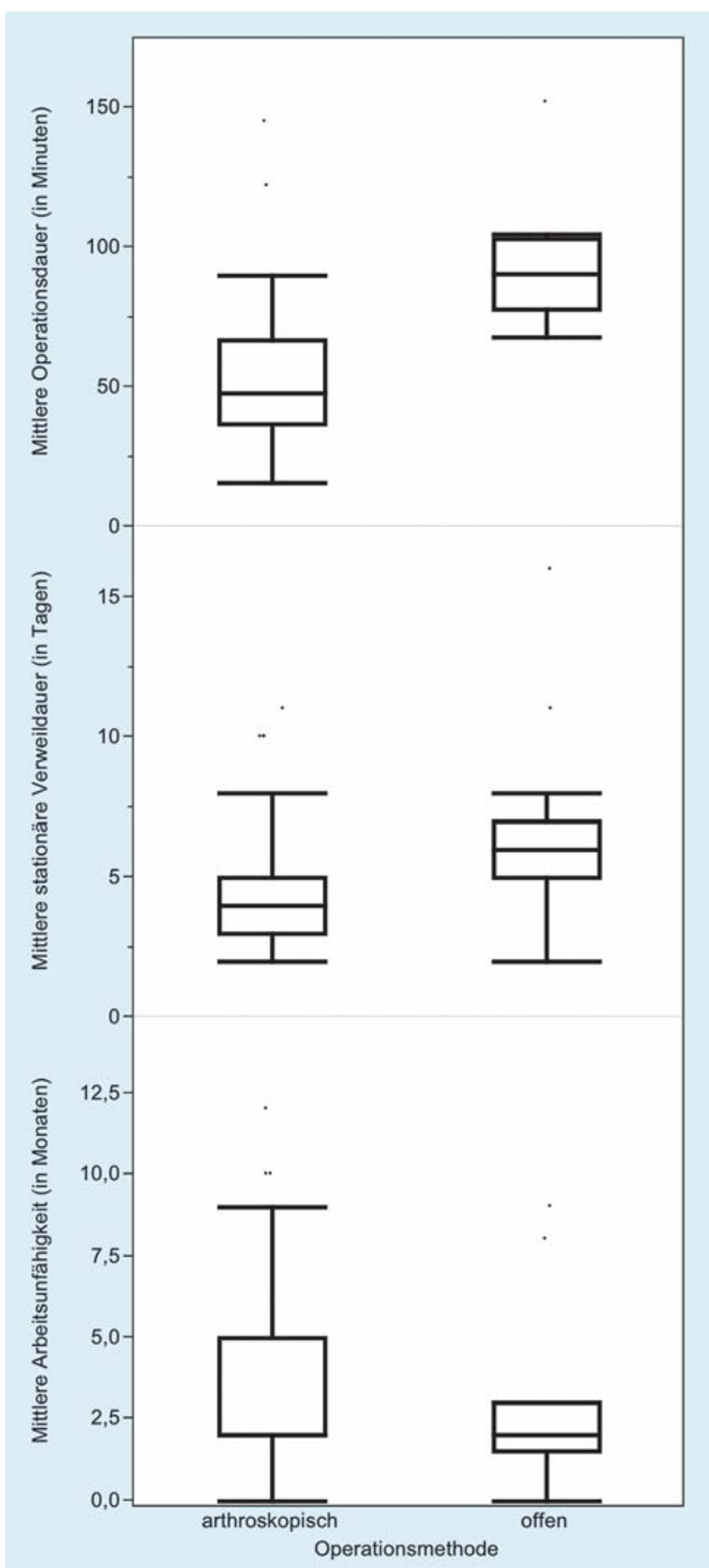

Abb. 4 Operationsdauer (in min), stationäre Verweildauer (in d) und Dauer der Arbeitsunfähigkeit in Abhängigkeit von der Operationsmethode (arthroskopisch vs. offen). 
Tab. 3 Ergebnisse der verschiedenen Operationsmethoden.

\begin{tabular}{|c|c|c|c|c|c|c|c|c|c|c|}
\hline $\begin{array}{l}\text { OP-Me- } \\
\text { thode }\end{array}$ & n & $\begin{array}{l}\text { Alter in } \\
\text { Jahren } \\
(\mathrm{MW} \pm \mathrm{SD})\end{array}$ & $\begin{array}{l}\text { Dauer bis zur } \\
\text { Nachunter- } \\
\text { suchung } \\
\text { in Monaten } \\
\text { (MW } \pm \text { SD) }\end{array}$ & $\begin{array}{l}\text { OP Dauer } \\
\text { in } \min \\
(\mathrm{MW} \pm \mathrm{SD})\end{array}$ & $\begin{array}{l}\text { Reluxations- } \\
\text { rate (n/in \%) }\end{array}$ & $\begin{array}{l}\text { stationäre } \\
\text { Verweildau- } \\
\text { er in Tagen } \\
\text { (SD } \pm \text { SD) }\end{array}$ & $\begin{array}{l}\text { Arbeitsun- } \\
\text { fähigkeit } \\
\text { (MW } \pm \mathrm{SD}) \\
\text { in Monaten }\end{array}$ & $\begin{array}{l}\text { UCLA Shoul- } \\
\text { der Score } \\
\text { (MW } \pm \text { SD) }\end{array}$ & $\begin{array}{l}\text { Rowe Score } \\
\text { (MW } \pm \text { SD) }\end{array}$ & $\begin{array}{l}\text { Constant } \\
\text { and Murley } \\
\text { Score } \\
\text { (MW } \pm \text { SD) }\end{array}$ \\
\hline arth. & 72 & $34,8 \pm 13,7$ & $43,4 \pm 18$ & $53,9 \pm 24,1$ & $8(11,1 \%)$ & $4,28 \pm 2,16$ & $3,3 \pm 2,52$ & $31,7 \pm 4,18$ & $85,8 \pm 23$ & $87,4 \pm 13,5$ \\
\hline \multirow[t]{2}{*}{ offen } & 21 & $45,1 \pm 14,2$ & $65 \pm 32,2$ & $94,3 \pm 23,8$ & $1(4,76 \%)$ & $6,3 \pm 2,34$ & $2,71 \pm 2,34$ & $31 \pm 4,31$ & $86,7 \pm 17,3$ & $82,1 \pm 16,3$ \\
\hline & & $\begin{array}{l}\mathrm{p}=0,0056^{*} \\
\text { (t-Test) }\end{array}$ & $\begin{array}{l}\mathrm{p}=0,0074^{*} \\
\text { (t-Test) }\end{array}$ & $\begin{array}{l}\mathrm{p}=0,0003^{*} \\
\text { (t-Test) }\end{array}$ & $\begin{array}{l}\mathrm{p}=0,39 \\
\text { (Chi-Quadrat, } \\
\text { Pearson) }\end{array}$ & $\begin{array}{l}p=0,0083^{*} \\
\text { (t-Test) }\end{array}$ & $\begin{array}{l}\mathrm{p}=0,37 \\
\text { (t-Test) }\end{array}$ & $\begin{array}{l}p=0,47 \\
\text { (t-Test) }\end{array}$ & $\begin{array}{l}p=0,86 \\
\text { (t-Test) }\end{array}$ & $\begin{array}{l}\mathrm{p}=0,19 \\
\text { (t-Test) }\end{array}$ \\
\hline
\end{tabular}

* = statistische Signifikanz

Tab. 4 Ergebnisse in Bezug auf die Arbeitsbelastung.

\begin{tabular}{|c|c|c|c|c|c|c|c|c|c|c|}
\hline REFA & $\mathbf{n}$ & $\begin{array}{l}\text { Alter in } \\
\text { Jahren } \\
\text { (MW } \pm \text { SD) }\end{array}$ & $\begin{array}{l}\text { Dauer bis zur } \\
\text { Nachunter- } \\
\text { suchung } \\
\text { in Monaten } \\
\text { (MW } \pm \text { SD) }\end{array}$ & $\begin{array}{l}\text { OP Dauer } \\
\text { in } \min \\
(\mathrm{MW} \pm \mathrm{SD})\end{array}$ & $\begin{array}{l}\text { Reluxations- } \\
\text { rate (n/in \%) }\end{array}$ & $\begin{array}{l}\text { stationäre } \\
\text { Verweildau- } \\
\text { er in Tagen } \\
\text { (SD } \pm \text { SD) }\end{array}$ & $\begin{array}{l}\text { Arbeitsun- } \\
\text { fähigkeit } \\
\text { (MW } \pm \mathrm{SD} \text { ) } \\
\text { in Monaten }\end{array}$ & $\begin{array}{l}\text { UCLA Shoul- } \\
\text { der Score } \\
\text { (MW } \pm \text { SD) }\end{array}$ & $\begin{array}{l}\text { Rowe Score } \\
(\mathrm{MW} \pm \mathrm{SD})\end{array}$ & $\begin{array}{l}\text { Constant } \\
\text { and Murley } \\
\text { Score } \\
\text { (MW } \pm \text { SD) }\end{array}$ \\
\hline $0-1$ & 49 & $38,1 \pm 15,8$ & $48,7 \pm 23,6$ & $66,2 \pm 32,5$ & $5(10,2 \%)$ & $4,06 \pm 1,8$ & $2,26 \pm 1,5$ & $32,6 \pm 3,35$ & $87,2 \pm 22,2$ & $88,5 \pm 13,5$ \\
\hline \multirow[t]{2}{*}{$2-4$} & 44 & $36,0 \pm 12,7$ & $47,8 \pm 23,9$ & $53,3 \pm 20,0$ & $4(9,1 \%)$ & $5,43 \pm 2,9$ & $4,18 \pm 2,9$ & $30,4 \pm 4,76$ & $84,7 \pm 21,4$ & $83,6 \pm 14,8$ \\
\hline & & $\begin{array}{l}p=0,4784 \\
\text { (t-Test) }\end{array}$ & $\begin{array}{l}\mathrm{p}=0,8531 \\
\text { (t-Test) }\end{array}$ & $\begin{array}{l}\mathrm{p}=0,062 \\
\text { (t-Test) }\end{array}$ & $\begin{array}{l}p=0,8561 \\
\text { (Chi-Quadrat, } \\
\text { Pearson) }\end{array}$ & $\begin{array}{l}\mathrm{p}=0,0091^{*} \\
\text { (t-Test) }\end{array}$ & $\begin{array}{l}\mathrm{P}=0,0006^{*} \\
\text { (t-Test) }\end{array}$ & $\begin{array}{l}p=0,0143^{*} \\
\text { (t-Test) }\end{array}$ & $\begin{array}{l}\mathrm{p}=0,5695 \\
\text { (t-Test) }\end{array}$ & $\begin{array}{l}\mathrm{p}=0,1021 \\
\text { (t-Test) }\end{array}$ \\
\hline
\end{tabular}

* = statistische Signifikanz

\section{Komplikationen}

Insgesamt erlitten 9 Patienten (9,7\%) eine Rezidivluxation. 18,3\% (17 von 93) aller Probanden konnten ihre ursprüngliche Tätigkeit nicht wieder aufnehmen ( 0 Tab. 5). 16 Patienten (17,2\%) begaben sich im weiteren Verlauf erneut in eine Behandlung wegen bestehender Restbeschwerden ohne spürbare Subluxationsphänomene anzugeben.

\section{Diskussion}

Mit einer Gesamtzahl von 93 an der Nachuntersuchung teilnehmenden Patienten lässt sich diese Studie mit bestehenden Arbeiten vergleichen. Auch die klinischen Ergebnisse der Scores liegen in einem mit anderen Studien vergleichbaren Bereich [7,8,10]. Vergleicht man die Ergebnisse nach offener und nach arthroskopischer Technik, so hat sich eine signifikant längere Operationsdauer bei der offenen Bankart-Operation gezeigt. Auch die stationäre Verweildauer war nach offener Operation signifikant länger. Wir haben nach offenem Bankart-Repair rein nominal eine deutlich geringere Reluxationsrate mit knapp 4,7\% im Vergleich zum arthroskopischen Vorgehen $(11,1 \%)$ festgestellt. Diese relativ geringe Reluxationsrate kann aufgrund der geringen Gruppengröße auch falsch niedrig sein. Der statistische Vergleich ergab keinen signifikanten Unterschied. Zieht man die Erfahrungen aus anderen Studien heran, so findet man Reluxationsraten von 3 bis $23 \%$, wobei die individuelle Belastung und Exposition die Reluxationsrate, bspw. durch Kontaktsportarten, erhöhen kann [26]. Diese Einflussmöglichkeiten sollten an dieser Stelle berücksichtigt werden. In anderen vergleichenden Studien wurden für die offene Versorgung Gruppengrößen von 22 bis 106 angegeben mit einer durchschnittlichen Reluxationsrate von 6,7\% [10]. Die ver- fahrensspezifischen Unterschiede (ALR vs. OLR) beschränken sich in unserer Analyse auf die Operations- und stationäre Verweildauer. Der größere Operationsaufwand beim offenen Vorgehen liegt auf der Hand und stellt keine Überraschung dar. Kürzere Operationszeiten zugunsten des arthroskopischen Vorgehens wurden durch zahlreiche Vorarbeiten bereits objektiviert [13]. Die kürzere stationäre Verweildauer nach ALR wurde bereits durch zahlreiche Studien beschrieben und lässt sich durch die geringere Invasivität und Schonung des Weichteilmantels bzw. der Subskapularissehne erklären [27].

Sozioökonomisch ergeben sich daraus für das arthroskopische Vorgehen bei vergleichbaren bzw. in unserem Kollektiv äquivalenten klinischen Ergebnissen deutliche Vorteile gegenüber der offenen Vorgehensweise [7,8,10,11]. Für besondere Fälle sollte die offene Technik als Alternative in Betracht gezogen werden. Insbesondere die Rekonstruktion von Rezidivluxationen ohne knöcherne Pfannendefekte oder Primäreingriffe bei Kontaktsportlern stellt ein solches Szenario dar [16,26]. Natürlich muss für Rezidivluxationen auch an die bewährten, technisch unterschiedlich realisierbaren Pfannenplastiken gedacht werden, wie bspw. der J-Span nach Resch, der Coracoid-Transfer (Latarjet) etc. $[28,29]$.

Wird der Einfluss unterschiedlicher Arbeitsbelastung unabhängig von der Operationsmethode analysiert, zeigten sich Unterschiede hinsichtlich der Operations- sowie der stationären Verweildauer, wobei kein statistisch signifikantes Niveau erreicht wurde. Während Patienten mit geringer körperlicher Arbeitsbelastung (REFA 0-1) eine im Mittel 13 min längere Operationszeit benötigen, ist die stationäre Verweildauer für Patienten mit REFA 2-4 im Mittel um 1,4 Tage länger im Vergleich zu Patienten mit REFA 0-1. Eine Erklärung für die längeren Operationszeiten war uns auch nach kritischer Durchsicht der Operationsprotokol- 
Tab. 5 Ergebnisse unter Berücksichtigung zur Arbeitsbelastung (REFA 0-1/REFA 2-4) und Operationsmethode.

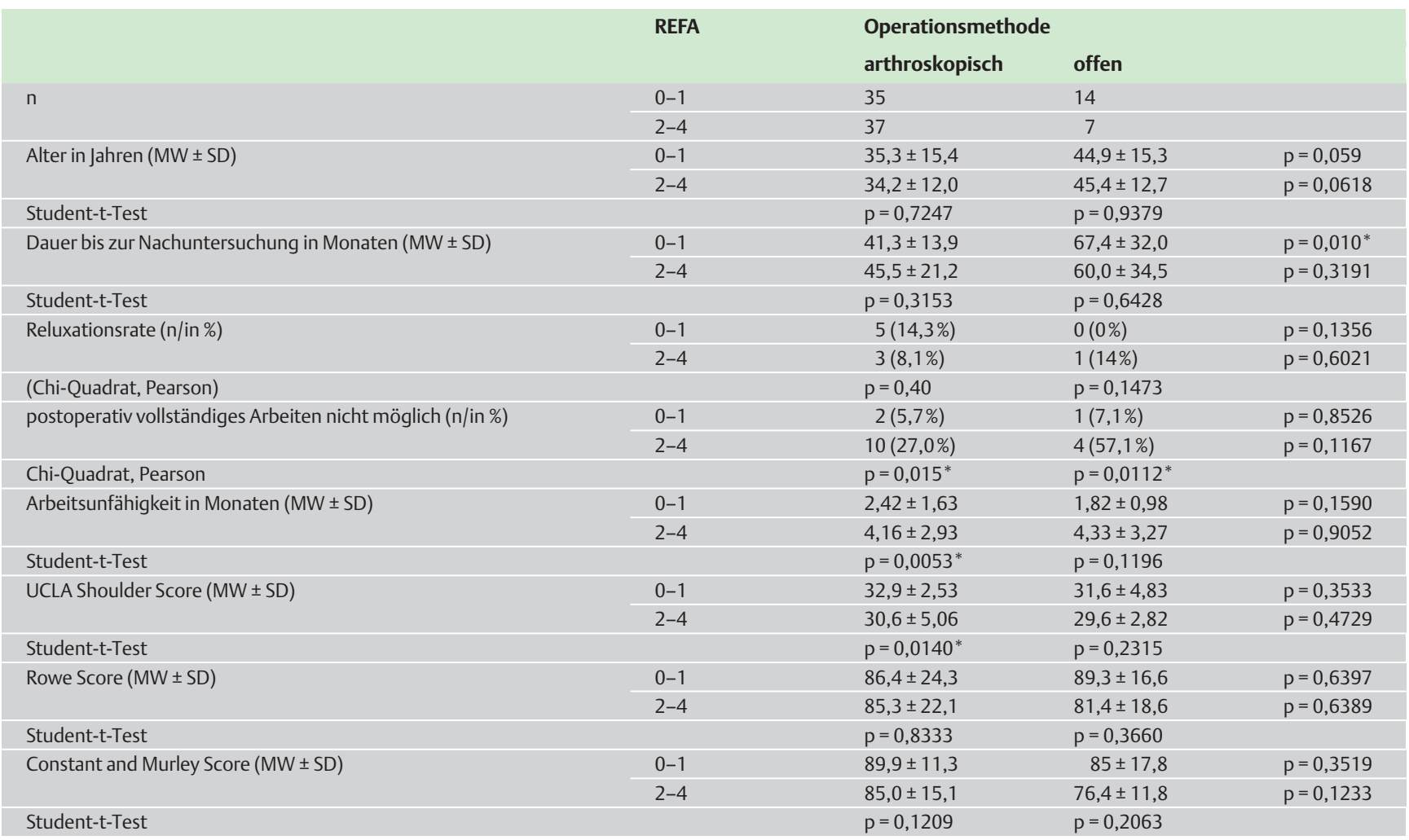

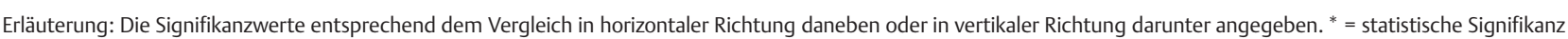

le nicht möglich. Die längere stationäre Verweildauer von Patienten aus den REFA-Gruppen 2-4 hingegen lässt sich möglicherweise durch belastungsbedingte Vorschäden durch repetitive Mikrotraumen ggf. erklären. Um diesen Aspekt differenziert zu analysieren, wären jedoch adäquate prospektive Studien notwendig. Interessanterweise hat der Altersunterschied zwischen den Gruppen „offen“ \pm (45 Jahre) und „arthroskopisch“ ( \pm 35 Jahre) keine Auswirkung auf das Outcome hinsichtlich der Funktionsscores. Dennoch muss dieser Unterschied in der Interpretation dieser Studie Beachtung finden. Da der Hauptfokus dieser Studie auf die Arbeitsfähigkeit in der ursprünglichen Tätigkeit lag, haben wir den Altersunterschied zur Kenntnis genommen und sehen dies als relative Einschränkung hinsichtlich der Vergleichbarkeit. Um dieses Dilemma zu lösen, wäre eine MatchedPair-Analyse notwendig, allerdings mit dem Nachteil einer geringeren Probandenzahl. Durch die Ausrichtung der Studie hinsichtlich der beruflichen Situation und Rehabilitation und der äquivalenten Funktionsscores nach ALR und OLR scheint sich die Altersdifferenz zwischen Mitte 30 und Mitte 40 Jahren wenig prominent auszuwirken.

Die Reluxationsrate im Gesamtkollektiv von 9,7\% ist vergleichbar mit den Erfahrungen anderer Autoren $[10,26]$. In unserem Patientenkollektiv fanden sich jedoch mehr Reluxationen unter den Patienten der Gruppe REFA 0-1 (14,3\%) als im schwer körperlich tätigen Kollektiv $(8,1 \%)$. Eine mögliche Erklärung für dieses Phänomen mag die kürzere Rehabilitationsdauer sein, möglicherweise eine insgesamt weniger austrainierte Statur und damit möglicherweise eine geringere muskuläre Führung des Schultergürtels. Dass ggf. auch muskelspezifische Veränderungen eine Rolle spielen, wurde erstmals durch Gamulin et al. erarbeitet [30]. Gamulin und Kollegen konnten erstmals zeigen, dass eine pathologische Verteilung der Muskelfasern bzw. histopathologische Vernarbungsprozesse des M. subscapularis zu einer signifikant erhöhten Reluxationsrate nach offener Bankart-Stabilisierung führen [30]. In unserem Kollektiv wurden hauptsächlich Berufstätige Mitte 30 bis Mitte 40 nachuntersucht. Für diese Altersklasse wurde unter den Freizeitsportlern der Begriff des „Weekend-Warriors“ geprägt. Der Weekend-Warrior beschreibt einen Freizeitsportler, der sich am Wochenende mehr oder weniger dem Freizeitsport widmet, jedoch aufgrund der Berufstätigkeit weniger trainiert ist. Diese Trainingsdefizite führen nachvollziehbarerweise vermehrt zu Verletzungen und betreffen insbesondere die Gruppe REFA 0-1. Es lässt sich jedoch keine klare bzw. belastbare Erklärung für die Differenz der Reluxationsraten herleiten.

Unsere Studienergebnisse können erstmals die bisher vermutete längere Arbeitsunfähigkeit von Patienten mit hoher körperlicher Arbeitsbelastung (REFA 2-4) gegenüber Patienten mit geringer körperlicher Arbeitsbelastung (REFA 0-1) sowohl für das offene wie arthroskopische Vorgehen objektivieren ( Tab. 5). Probanden der Gruppe REFA 2-4 benötigten einen ungefähr doppelt so langen Zeitraum für die Rehabilitation mit ca. 4 Monaten gegenüber ca. 2 Monaten für Probanden der Gruppe REFA 0-1, um Arbeitsfähigkeit zu erlangen. Diese kürzeren bzw. längeren Arbeitsunfähigkeiten hatten allerdings keinen Einfluss auf die dauerhafte Stabilität bzw. die Reluxationsrate. Die Zusammenfassung der REFA-Gruppen 0-1 sowie 2-4 haben wir in dieser Studie aufgrund des Belastungssprungs zwischen der REFA-Gruppe 1 und 2 vorgenommen und der relativ geringen Gruppenstärke nach OLR. Eine sinnvolle Unterteilung in 3 Untergruppen wäre durchaus eine Alternative gewesen, wobei die Gruppenstärke nach OLR eine sinnvolle Interpretation der Daten zu stark limitiert hätte. 
Eine Limitierung der Anwendbarkeit besteht nicht. Bisher wurde diese etablierte Klassifikation nur selten genutzt [31]. Die Rate derer, die ihre ursprüngliche Tätigkeit nicht wieder aufnehmen konnten, betrug nach ALR 16,7\% (12 aus 72) und nach OLR 23,8\% (5 aus 21) mit einem Vorteil für das arthroskopische Vorgehen, ohne jedoch statistisch signifikant zu sein. Dieser Prozentsatz ist aus unserer Sicht unter Berücksichtigung der bisherigen Analysen mit Berücksichtigung der Sportfähigkeit überraschend hoch [10,26]. Da diese Ergebnisse nicht mit anderen Arbeiten in Bezug gesetzt werden können, bedarf es weiterer Studien, die diese Zusammenhänge prüfen. Der Vergleich zwischen Patienten mit gleicher Arbeitsbelastung (sowohl REFA 0-1 als auch REFA 24) und unterschiedlicher Operation (ALR vs. OLR) ergab dagegen keinen signifikanten Unterschied.

Die vorliegende Studie hat Schwächen aufgrund der retrospektiven Patientenrekrutierung, der unterschiedlich großen Gruppenstärken sowie aufgrund des Altersunterschieds in den Gruppen ALR und OLR.

Die standardisierte Operationstechnik und identische Nachbehandlung sowie die klaren Ein- und Ausschlusskriterien geben unseres Erachtens jedoch eine ausreichende Bewertungsbasis, um die hier erhobene Fragestellung zu bearbeiten.

\section{Schlussfolgerungen \\ $\nabla$}

Die offene und arthroskopische Kapsel-Labrum-Rekonstruktion ermöglichen in Bezug auf die Arbeitsunfähigkeitsdauer äquivalente Ergebnisse. In unserem Vorgehen waren die verfahrensspezifischen klinischen Resultate vergleichbar. Für das arthroskopische Vorgehen haben wir signifikant kürzere Operationszeiten und stationäre Verweildauern festgestellt.

Die Hypothese, dass beruflich belastende Tätigkeiten mit einer verlängerten Arbeitsunfähigkeitsdauer einhergehen, wurde bestätigt. Die Arbeitsbelastung hatte hingegen in unserem Vorgehen keinen Einfluss auf das postoperative klinische Outcome unabhängig davon, ob arthroskopisch oder offen rekonstruiert wurde. Die Rate von ca. 18,3\% (17 aus dem Gesamtkollektiv mit 93 Fällen) beruflich nicht rehabilitierbarer Patienten ist hoch und bedarf einer weiteren Verbesserung.

Um diese sozioökonomisch relevanten Auswirkungen zu verbessern, sind weitere prospektiv angelegte, verfahrensspezifische Studien notwendig, die ggf. weiterentwickelte Operationstechniken prüfen.

\section{Interessenkonflikt: Nein}

\section{Literatur}

1 Zacchilli MA, Owens BD. Epidemiology of shoulder dislocations presenting to emergency departments in the United States. J Bone Joint Surg Am 2010; 92: 542-549

2 Boone JL, Arciero RA. Management of failed instability surgery: how to get it right the next time. Orthop Clin North Am 2010; 41: 367-379

3 Antonio GE, Griffith JF, Yu AB et al. First-time shoulder dislocation: High prevalence of labral injury and age-related differences revealed by MR arthrography. J Magn Reson Imaging 2007; 26: 983-991

4 Longo UG, Loppini M, Rizzello G et al. Glenoid and humeral head bone loss in traumatic anterior glenohumeral instability: a systematic review. Knee Surg Sports Traumatol Arthrosc 2014; 22: 392-414

5 Taylor DC, Arciero RA. Pathologic changes associated with shoulder dislocations. Arthroscopic and physical examination findings in firsttime, traumatic anterior dislocations. Am J Sports Med 1997; 25: 306-311

6 Bankart AS. The pathology and treatment of recurrent dislocation of the shoulder joint. Br J Surg 1938; 26: 23-29
7 Cole BJ, L'Insalata J, Irrgang J et al. Comparison of arthroscopic and open anterior shoulder stabilization. A two to six-year follow-up study. J Bone Joint Surg Am 2000; 82: 1108-1114

8 Zaffagnini S, Marcheggiani Muccioli GM, Giordano G et al. Long-term outcomes after repair of recurrent post-traumatic anterior shoulder instability: comparison of arthroscopic transglenoid suture and open Bankart reconstruction. Knee Surg Sports Traumatol Arthrosc 2012; 20: 816-821

9 Rowe CR, Patel D, Southmayd WW. The Bankart procedure: a long-term end-result study. J Bone Joint Surg Am 1978; 60: 1-16

10 Petrera $M$, Patella V, Patella $S$ et al. A meta-analysis of open versus arthroscopic Bankart repair using suture anchors. Knee Surg Sports Traumatol Arthrosc 2010; 18: 1742-1747

11 Harris JD, Gupta AK, Mall NA et al. Long-term outcomes after bankart shoulder stabilization. Arthroscopy 2013; 29: 920-933

$12 \mathrm{Kim} \mathrm{SH}, \mathrm{Ha} \mathrm{KI}, \mathrm{Cho}$ YB et al. Arthroscopic anterior stabilization of the shoulder: two to six-year follow-up. J Bone Joint Surg Am 2003; 85: $1511-1518$

13 Bottoni CR, Smith EL, Berkowitz MJ et al. Arthroscopic versus open shoulder stabilization for recurrent anterior instability: a prospective randomized clinical trial. Am J Sports Med 2006; 34: 1730-1737

14 Martetschlager F, Kraus TM, Hardy P et al. Arthroscopic management of anterior shoulder instability with glenoid bone defects. Knee Surg Sports Traumatol Arthrosc 2013; 21: 2867-2876

15 Scheibel M, Kraus N, Diederichs $G$ et al. Arthroscopic reconstruction of chronic anteroinferior glenoid defect using an autologous tricortical iliac crest bone grafting technique. Arch Orthop Trauma Surg 2008; 128: $1295-1300$

16 Cho NS, Yi JW, Lee BG et al. Revision open Bankart surgery after arthroscopic repair for traumatic anterior shoulder instability. Am J Sports Med 2009; 37: 2158-2164

17 Millett PJ, Clavert P, Warner JJ. Open operative treatment for anterior shoulder instability: when and why? J Bone Joint Surg Am 2005; 87: 419-432

18 Owens BD, DeBerardino TM, Nelson BJ et al. Long-term follow-up of acute arthroscopic Bankart repair for initial anterior shoulder dislocations in young athletes. Am J Sports Med 2009; 37: 669-673

19 Brophy RH, Gill CS, Lyman S et al. Effect of shoulder stabilization on career length in national football league athletes. Am J Sports Med 2011; 39: 704-709

20 Baker CL 3rd, Mascarenhas R, Kline AJ et al. Arthroscopic treatment of multidirectional shoulder instability in athletes: a retrospective analysis of 2- to 5-year clinical outcomes. Am J Sports Med 2009; 37: 1712-1720

21 Stein T, Linke RD, Buckup J et al. Shoulder sport-specific impairments after arthroscopic Bankart repair: a prospective longitudinal assessment. Am J Sports Med 2011; 39: 2404-2414

22 Jobe FW, Giangarra CE, Kvitne RS et al. Anterior capsulolabral reconstruction of the shoulder in athletes in overhand sports. Am J Sports Med 1991; 19: 428-434

23 Larrain MV, Montenegro HJ, Mauas DM et al. Arthroscopic management of traumatic anterior shoulder instability in collision athletes: analysis of 204 cases with a 4- to 9-year follow-up and results with the suture anchor technique. Arthroscopy 2006; 22: 1283-1289

24 Amstutz HC, Sew Hoy AL, Clarke IC. UCLA anatomic total shoulder arthroplasty. Clin Orthop Relat Res 1981; 155: 7-20

25 Constant CR, Murley AH. A clinical method of functional assessment of the shoulder. Clin Orthop Relat Res 1987; 214: 160-164

26 Schrumpf MA, Maak TG, Delos D et al. The management of anterior glenohumeral instability with and without bone loss: AAOS exhibit selection. J Bone Joint Surg Am 2014; 96: e12

27 Green MR, Christensen KP. Arthroscopic versus open Bankart procedures: a comparison of early morbidity and complications. Arthroscopy 1993; 9: 371-374

28 Lafosse L, Boyle S. Arthroscopic Latarjet procedure. J Shoulder Elbow Surg 2010; 19: 2-12

29 Tischer T, Vogt S, Kreuz PC et al. Arthroscopic anatomy, variants, and pathologic findings in shoulder instability. Arthroscopy 2011; 27: 1434-1443

30 Gamulin A, Dayer R, Lubbeke A et al. Primary open anterior shoulder stabilization: a long-term, retrospective cohort study on the impact of subscapularis muscle alterations on recurrence. BMC Musculoskelet Disord 2014; 15: 45

31 Schroter S, Mueller J, van Heerwaarden $R$ et al. Return to work and clinical outcome after open wedge HTO. Knee Surg Sports Traumatol Arthrosc 2013; 21: 213-219 